\title{
Soliton Microcombs in Integrated Chalcogenide Microresonators
}

\section{Di Xia}

Sun Yat-Sen University

\section{Zelin Yang}

Sun Yat-Sen University

\section{Pingyang Zeng}

Sun Yat-Sen University

Bin Zhang ( $\nabla$ zhangbin5@mail.sysu.edu.cn )

Sun Yat-sen University

Jiayue Wu

Sun Yat-Sen University

Zifu Wang

Sun Yat-Sen University

Jiaxin Zhao

Sun Yat-Sen University

Mingqi Gao

Sun Yat-Sen University

Yufei Huang

Sun Yat-Sen University

Jianteng Huang

Sun Yat-Sen University

Liyang Luo

Sun Yat-Sen University

Dong Liu

Sun Yat-sen University

\section{Shuixian Yang}

Sun Yat-Sen University

\section{Hairun Guo}

Shanghai University https://orcid.org/0000-0002-9913-2817

Zhaohui Li

Sun Yat-Sen University 
Keywords:

Posted Date: February 3rd, 2022

DOI: https://doi.org/10.21203/rs.3.rs-1322946/v1

License: (c) (1) This work is licensed under a Creative Commons Attribution 4.0 International License. Read Full License 
3 Di Xia ${ }^{1,5}$, Zelin Yang ${ }^{1,5}$, Pingyang Zeng ${ }^{1,5}$, Bin Zhang ${ }^{1,2 *}$, Jiayue Wu ${ }^{1}$, Zifu Wang ${ }^{1}$, Jiaxin

4 Zhao ${ }^{1}$, Mingqi Gao ${ }^{1}$, Yufei Huang ${ }^{1}$, Jianteng Huang ${ }^{1}$, Liyang Luo ${ }^{1}$, Dong Liu ${ }^{1}$, Shuixian

$5 \quad$ Yang ${ }^{1}$, Hairun $\mathrm{Guo}^{3}$, and Zhaohui $\mathrm{Li}^{1,2,4, *}$

6

7

${ }^{1}$ Guangdong Provincial Key Laboratory of Optoelectronic Information Processing Chips and Systems, School of Electrical and Information Technology, Sun Yat-sen University, Guangzhou, 510275, China.

${ }^{2}$ Key Laboratory of Optoelectronic Materials and Technologies, Sun Yat-sen University, Guangzhou, 510275, China.

${ }^{3}$ Key Laboratory of Specialty Fiber Optics and Optical Access Networks, Shanghai University, Shanghai, 200444, China.

${ }^{4}$ Southern Marine Science and Engineering Guangdong Laboratory (Zhuhai), Zhuhai, 519000, China.

${ }^{5}$ These authors contributed equally: Di Xia, Zelin Yang, Pingyang Zeng

*e-mail: zhangbin5@mail.sysu.edu.cn, 1zhh88@mail.sysu.edu.cn

\section{Abstract:}

Photonic integrated microcombs have enabled advanced applications in optical communication, microwave synthesis, and optical metrology, which in nature unveil an optical dissipative soliton pattern under cavity-enhanced nonlinear processes. The most decisive factor of microcombs lies in the photonic material platforms, where materials with high nonlinearity and in capacity of high-quality chip integration are highly demanded. In this work, we present a home-developed chalcogenide glasses- $\mathrm{Ge}_{25} \mathrm{Sb}_{10} \mathrm{~S}_{65}(\mathrm{GeSbS})$ for the nonlinear photonic integration and for the dissipative soliton microcomb generation. Compared with the current integrated nonlinear platforms, the GeSbS features wider 
transparency from the visible to $11-\mu \mathrm{m}$ region, stronger nonlinearity, and lower thermorefractive coefficient, and is CMOS compatible in fabrication. In this platform, we achieve chip-integrated optical microresonators with a quality (Q) factor above $2 \times 10^{6}$, and carry out lithographically controlled dispersion engineering. In particular, we demonstrate that both a bright soliton-based microcomb and a dark-pulsed comb are generated in a single microresonator, in its separated fundamental polarized mode families under different dispersion regimes. The overall pumping power is on the ten-milliwatt level, determined by both the high Q-factor and the high material nonlinearity of the microresonator. Our results may contribute to the field of nonlinear photonics with an alternative material platform for highly compact and high-intensity nonlinear interactions, while on the application aspect, contribute to the development of soliton microcombs at low operation power, which is potentially required for monolithically integrated optical frequency combs. 


\section{Introduction}

Integrated nonlinear photonics have combined nonlinear optics with state-of-the-art photonic integration and have unprecedentedly promoted light-matter interactions in terms of efficiency, bandwidth, and coherence ${ }^{1-6}$. They have enabled revolutionary techniques including chip-integrated optical frequency combs (OFCs $)^{5,7}$, ultra-high bandwidth electrooptical modulation ${ }^{2}$, and chip-scale quantum optics ${ }^{8}$. Of particular interest is the dissipative soliton-based OFCs in photonic integrated microresonators, which can be generated at a low pump power ${ }^{9}$ and have a broad bandwidth with fully coherent laser lines, benefiting from cavity-enhanced nonlinear efficiency and the lithographically controlled dispersion engingeering ${ }^{7}$. To date, they have enabled various advanced applications, including massive parallel optical telecommunication ${ }^{10-12}$, low-noise microwave synthesis ${ }^{13}$, parallel LiDAR $^{14}$, ${ }^{15}$, photonic neuromorphic computing ${ }^{16}$, and other photonic functionalities ${ }^{3,17,18}$ for chipscale time and frequency measurements.

Tremendous nonlinear material platforms have been developed to realize highperformance soliton microcombs ${ }^{7}$. Recent advances have witnessed several material platforms that could successfully support integrated soliton microcombs, such as silicon nitride $\left(\mathrm{Si}_{3} \mathrm{~N}_{4}\right)^{6,19,20}$, high-index doped silica $(\mathrm{Hydex})^{11,21}$, aluminum nitride $(\mathrm{AlN})^{22,23}$, gallium nitride $(\mathrm{GaN})^{24}$, lithium niobate $\left(\mathrm{LiNbO}_{3}\right)^{25-27}$, silicon carbide $(\mathrm{SiC})^{8,28}$ as well as $\mathrm{AlGaAs}^{9,29}$ on insulator. Amorphous $\mathrm{Si}_{3} \mathrm{~N}_{4}$ seems to be particularly fruitful in soliton combs, which has wide transparency, free from two-photon absorption (TPA), higher nonlinearity $\left(2.4 \times 10^{-19} \mathrm{~m}^{2} / \mathrm{W}\right)$ than Hydex, and has supported record-high quality (Q) factors in integrated microresonators ${ }^{6}$. On another aspect, semiconductor platforms such as AlGaAs 
on insulator could introduce even higher nonlinearity to reduce the pump power of soliton combs to sub-milliwatt $(\mathrm{mW})$ level ${ }^{9}$. Crystalline-based platforms including AlN and $\mathrm{LiNbO}_{3}$ introduce extra quadratic nonlinearities to generate the ultra-broadband soliton combs, which are potentially required for the self-referencing of microcombs ${ }^{22,} 30$. Particularly, most recently, monolithic microcomb chips have been developed based on the hybrid integration of such high-Q microresonators with III-V lasing chips, which are prone to be fully integrated in realistic systems $\mathrm{s}^{31,32}$.

Yet, limitations are also realized in operating such platforms for soliton combs with low pump power, compact and flexibility in high volume microcomb-chip fabrication. For instance, additional processes in the fabrication of $\mathrm{Si}_{3} \mathrm{~N}_{4}$ thick films for bright soliton microcombs are required, including crack-mitigation, chemical-mechanical polishing, and high temperature anneals (exceeding $1100^{\circ} \mathrm{C}$ ) strategies ${ }^{33,34}$. Crystalline platforms require the wafer-bonding process to be integrated on insulator substrates. In this way, the flexibility of performing dispersion engineering is reduced, and the yield of devices may be degraded as well ${ }^{6,35}$. Additionally, semiconductor resonators usually feature a high thermorefractive coefficient (TOC), which may hinder the existence of soliton combs ${ }^{24,29}$. As such, there exists a continuous motivation of seeking advanced material platforms, which could patch up the above issues and can be alternative to present platforms. In the meantime, while the current soliton microcombs are mostly designed and operated in the telecom band, it is equally essential for the material to open access in new wavelength regions for a broader range of microcomb applications, such as precise spectroscopy in the mid-infrared $(\mathrm{MIR})^{36}$ 

platform for soliton microcomb generation in high-quality and chip-scale microresonators. The material is $\mathrm{Ge}_{25} \mathrm{Sb}_{10} \mathrm{~S}_{65}(\mathrm{GeSbS})$ that inherits the ultrabroad transmission window with absence of TPA, large refractive index and Kerr nonlinearity ${ }^{37-42}$ of ChGs, and shows flexibility in photonic integration on silicon-based chips $^{43-45}$. In the meantime, with modified compounds, GeSbS could overcome existing problems of As-based ChGs, and features an improved laser damage threshold (LDT) with a reduced TOC. We fabricate chipintegrated GeSbS microresonators with an intrinsic Q-factor above $2 \times 10^{6}$, and demonstrate the generation of both the bright dissipative soliton microcombs and the dark pulse microcombs. In particular, the two microcomb regimes can be implemented in a single microresonator in the two fundamental polarized mode families that are lithographically engineered to induce normal and anomalous dispersive effects. Given a high Q-factor and high nonlinearity of the microresonator, the soliton comb is supported with a low pump power on the $10-\mathrm{mW}$ level, which is prone to implementing monolithic frequency comb chips for integrated metrology applications, such as compact dual-comb spectrometers and MIR spectroscopy. 

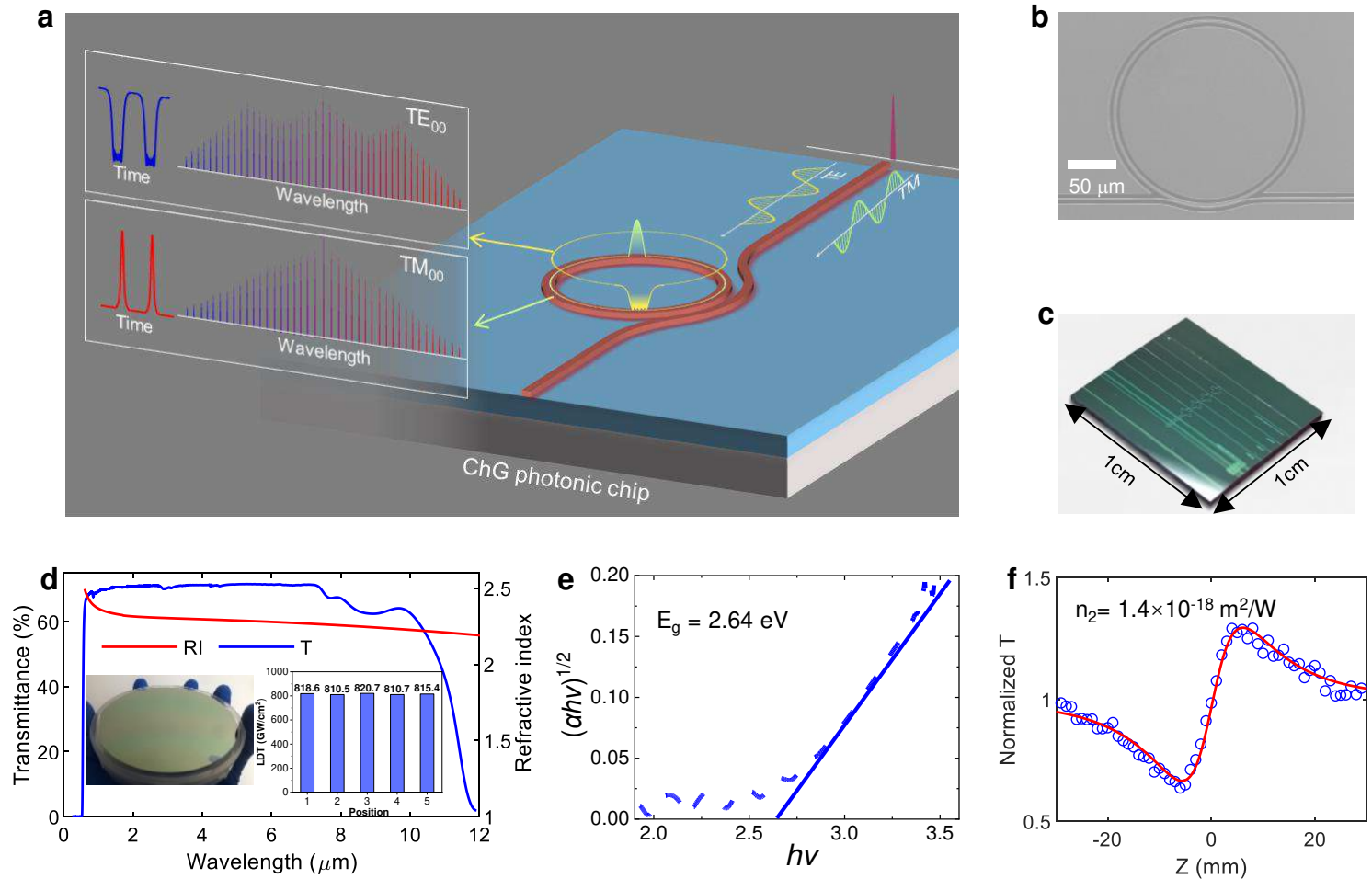

Fig.1 Nonlinear Chalcogenide photonic chip for soliton microcomb. (a) Schematic illustration of soliton microcomb generations in an integrated $\mathrm{ChG}$ microresonator, including dark and bright soliton combs in the $\mathrm{TE}_{00}$ and TM0o modes. (b) Scanning electron microscopy (SEM) image of a GeSbS microresonator with a radius of 100 $\mu \mathrm{m}$ with an integrated pulley bus waveguide. (c) Photograph of a fabricated $1 \mathrm{~cm} \times 1 \mathrm{~cm}$ GeSbS photonic chip comprises several microresonators with different structural parameters. (d) Measured transparency window and refractive index of the GeSbS bulk material. Inset: photograph of the GeSbS film (left) and the measured LDT at 5 different positions of the film using the femtosecond pulse laser(see materials and methods part)r, respectively (right). (e) Measured Tauc's plot for bandgap determination of GeSbS film. (f) Z-scan (close aperture) trace for nonlinear

\section{Results}

Nonlinear GeSbS photonics for soliton microcomb generation.

Figure 1a illustrates the schematic of $\mathrm{ChG}$ photonic chip-based soliton microcomb 

substrates by thermal evaporation at a low temperature (less than $\left.350{ }^{\circ} \mathrm{C}\right)^{24}$. The material also features a high laser damage threshold (ca. $820.7 \mathrm{GW} / \mathrm{cm}^{2}$ ), strong Kerr nonlinearity $\left(1.4 \times 10^{-18} \mathrm{~m}^{2} / \mathrm{W} \text { at } 1550 \mathrm{~nm}\right)^{46}$, large linear index $\left(\mathrm{n}_{0} \sim 2.2\right)$, large bandgap $(2.64 \mathrm{eV})$, optic coefficient (ca. $3.1 \times 10^{-5} / \mathrm{K}$, see Fig. 4a).
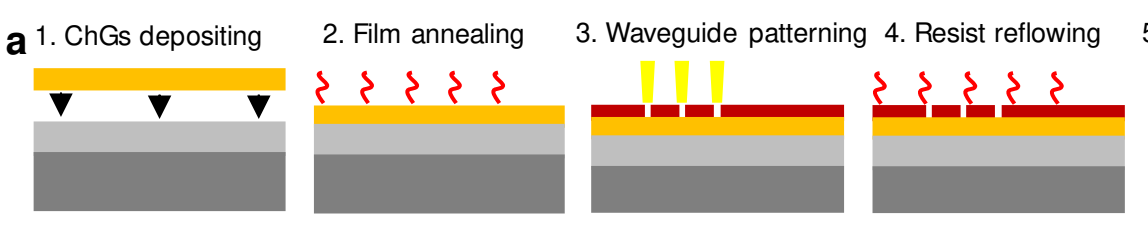

5. Optimized dry etching
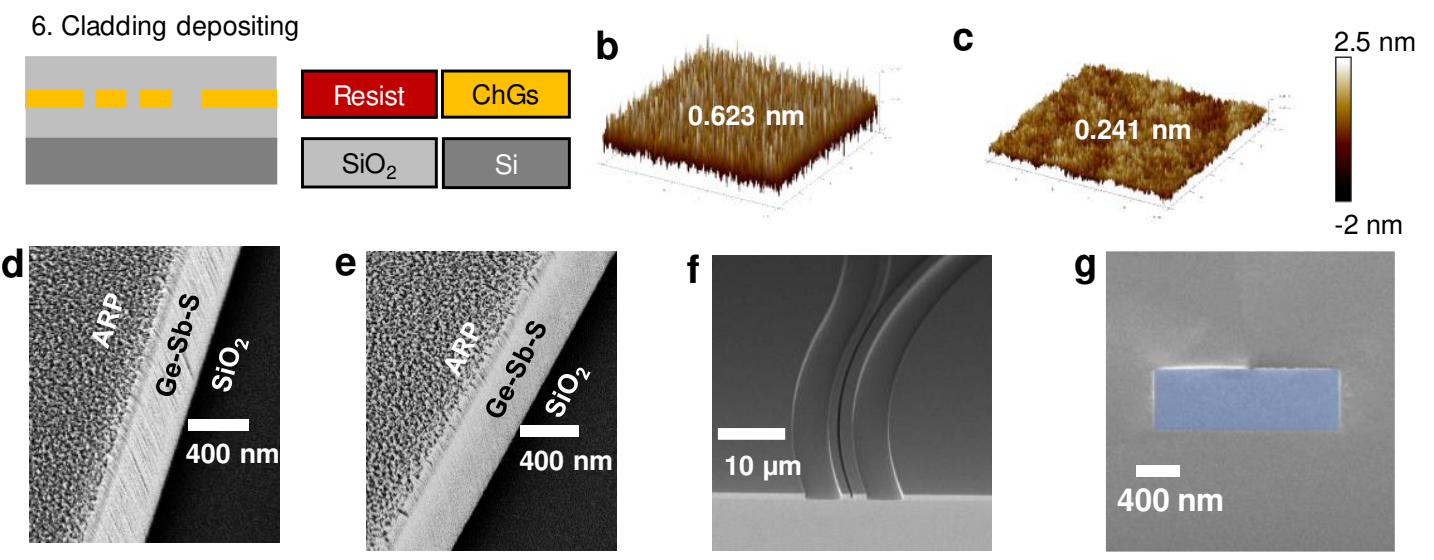

Fig.2 Fabrication of high Q-factor GeSbS microresonators. (a) Fabrication processes for achieving high Q integrated microresonators. (b), (c) 3D Atomic-force microscopy (AFM) images of GeSbS film in $5 \times 5 \mu \mathrm{m}^{2}$ area onchip before and after the annealing process at $350{ }^{\circ} \mathrm{C}$. (d-g) SEM images of the sidewall of the waveguides before and after the improved dry etching process, the pulley coupling structure, and the cross-section with a silica cladding waveguide, respectively. In (f), a compact photonic chip was demonstrated with the optimized coupling efficiency of $-3.5 \mathrm{~dB}(45 \%)$ per facet at $1550 \mathrm{~nm}$ using inverse taper couplers at the chip edge.

Fabrication of high $Q$-factor GeSbS microresonators. dramatic material properties for nonlinear optics applications ${ }^{9}$. The surface roughness is 
critical for achieving high $Q$-factor photonic integrated devices ${ }^{34}$. An improved waveguide fabrication process was provided to minimize the surface roughness of the top and sidewall of the microresonators, including optimized thermal-annealing of $\mathrm{ChG}$ film, thermalrefluxing the patterned mask-layer, transferring the pattern by an optimized plasma etching process, see Fig. 2a. The thermal annealing process is an effective and straightforward method to remove the surface roughness of amorphous GeSbS film. The root-mean-squared (RMS) roughness is decreased from $0.62 \mathrm{~nm}$ of the as-deposited film to $0.24 \mathrm{~nm}$ of the film annealed at $350^{\circ} \mathrm{C}$ correspondingly. Moreover, we focused on reducing scattering loss from in-situ polymer formation on the sidewall of waveguides by optimizing the dry etching process ${ }^{16}$. An optimized $\mathrm{CF}_{4} / \mathrm{CHF}_{3} / \mathrm{Ar}$ gas-based reactive ion etching and inductively coupled plasma (RIE-ICP) etching recipe was successfully achieved for smoother sidewalls of the microresonator by adjusting the flow of $\mathrm{O}_{2}$ and $\mathrm{CF}_{4}$ gases ${ }^{34,46}$, see Fig. $2 \mathbf{d}$ and $2 \mathbf{e}$. Based on the abovementioned processes, the top and sidewall surfaces of the microresonator could still be smooth after the resist removing, see Fig. 2f. As a result, a $Q-$ factor of more than $10^{6}$ was achieved, in contrast to the $Q$-factor of GeSbS microresonator with the same geometric dimensioning using the previously reported RIE-ICP etching recipe was only $10^{5}$ level $^{47}$. Moreover, the cross-section of the waveguide presents an almost vertical sidewall (see Fig. 2g), which is beneficial for accurate geometry dispersion control. 

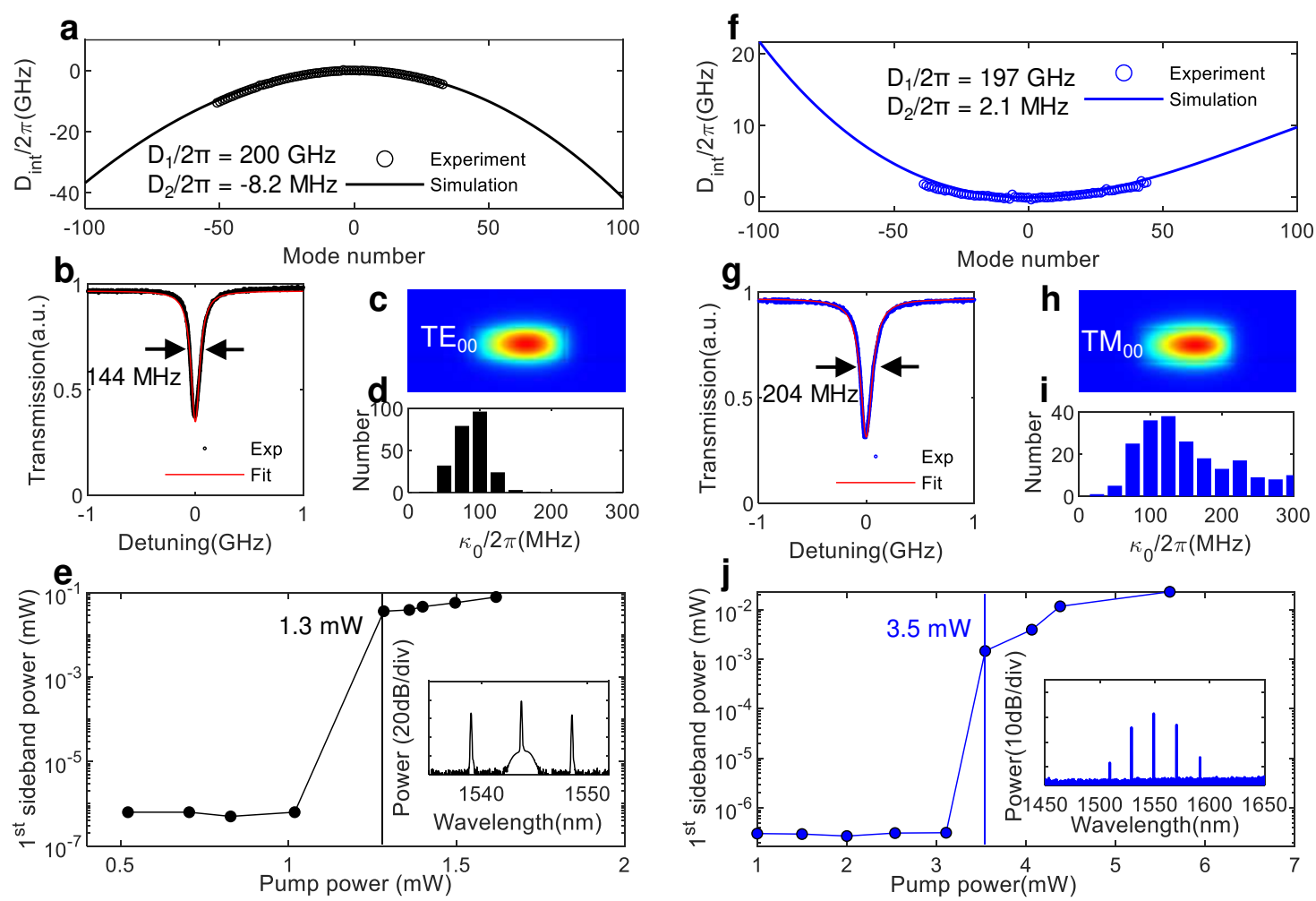

Fig.3 Characterization of high $Q$-factor GeSbS microresonators with TE $E_{00}$ resonances (a-e) and $\mathrm{TM}_{00}$ resonances (f - j), respectively. (a), (f) The calculated and measured resonator dispersion for $\mathrm{TE}_{00}$ and $\mathrm{TM}_{00}$ modes with a radius of $100 \mu \mathrm{m}$, and the cross-section is $2.4 \mu \mathrm{m} \times 0.8 \mu \mathrm{m}$ (width $\times$ height), respectively. The measured FSR $\left(\mathrm{D}_{1} / 2 \pi\right)$ is $200 \mathrm{GHz}, 197 \mathrm{GHz}$, fitted GVD $\left(\mathrm{D}_{2} / 2 \pi\right)$ is $-8.2 \mathrm{MHz}, 2.1 \mathrm{MHz}$ for TE00, TM00 mode, respectively. (b), (g) The measured transmission spectra at $1556 \mathrm{~nm}$ and $1555 \mathrm{~nm}$, revealing full-width-at-half-maximum (FWHM) to be $144 \mathrm{MHz}$ and $204 \mathrm{MHz}$ from the Lorentz fitting curves. (c), (h) the corresponding mode profiles. (d), (i) Histogram of intrinsic linewidths. (e), (j) The output power of the first generated FWM sideband as a function of input power. Insets: the measured output optical spectra, showing 3-FSR (FSR $=200 \mathrm{GHz})$ and 16-FSR $(\mathrm{FSR}=197$ $\mathrm{GHz}$ ) spaced $1^{\text {st }}$ sidebands for or TE 00 and TM00 modes, respectively.

\section{Characterization of high $Q$-factor GeSbS microresonators.}

Next, we systematically studied the dispersion, Q-factors, and OPO threshold powers based on GeSbS microresonators ${ }^{48}$. To further ensure accurate dispersion control of the waveguide, refractive index variation during the annealing process was also considered in device design, as shown in SI Fig. S1. Normal material GVD was exhibited in the telecom band (ca. $-400 \mathrm{ps} / \mathrm{nm} / \mathrm{km}$ at $1550 \mathrm{~nm}$ ) by measuring the linear indices of GeSbS film, which had to tailor the waveguide dimensions to achieve strongly anomalous geometric GVD. In 
the context of microresonators, the dispersion can lead to a deviation of resonances from equidistant mode spacing. The integrated dispersion $\mathrm{D}_{\text {int }}$ can express ${ }^{49}$ :

$$
D_{\text {int }}=\omega_{\mu}-\omega_{0}-D_{1} \mu=\frac{D_{2} \mu^{2}}{2 !}+\frac{D_{3} \mu^{3}}{3 !}+\sum_{m>3} \frac{D_{m} \mu^{m}}{m !}
$$

where $\mu, \omega_{\mu}$, and $\mathrm{m}$ are the relative mode numbers, angular frequencies of the resonances, and a positive integer, respectively. We experimentally measured the frequency difference using a fiber ring resonator and a fiber loop ${ }^{21}$. By analyzing the resonance variation from equidistant mode spacing, the $\mathrm{D}_{2} / 2 \pi$ of $\mathrm{TE}_{00}$ and $\mathrm{TM}_{00}$ mode are $-8.2 \mathrm{MHz}$ and $2.1 \mathrm{MHz}$ at the center frequency of around 193.4 THz, see Fig. 3a and 3f. Considering the material dispersion of $\mathrm{GeSbS}$ and $\mathrm{SiO}_{2}$, the microresonator dispersion as a function of relative mode numbers was also calculated by means of the finite element method (see Materials and methods). The simulated integrated dispersion profiles for both the $\mathrm{TE}_{00}$ and the $\mathrm{TM}_{00}$ mode families are shown in Fig. 3a and 3f, which have a good agreement with the experimental measurements. The fluctuations of the thickness (ca. $\pm 6.5 \mathrm{~nm}$ ) and refractive index (ca. \pm 0.0075 ) of the 4-inch ChG film are also considered in precise GVD control (see SI Fig. S1).

The intrinsic $Q$-factor of our microresonators is typically above $10^{6}$ for both mode families, see Fig. 3b and 3g. In a number of microresonator samples on the same fabrication batch, all the resonances within the measurement range (1510-1630 nm) are characterized, which show a mean value of $Q$-factor of ca. $1.97 \times 10^{6}$ for the $\mathrm{TE}_{00}$ mode family and ca. $1.38 \times 10^{6}$ for TM $\mathrm{M}_{00}$ mode family, see Fig. 3d and 3i. The pump threshold for OPO in such high-Q microresonators was also characterized by measuring the output powers of the first generated FWM sidebands with different input power, which for a pair of selected $\mathrm{TE}_{00}$ and 
TM 00 modes are $1.3 \mathrm{~mW}$ and $3.5 \mathrm{~mW}$, respectively. Moreover, in a $20 \mu \mathrm{m}$-radius microresonator, the measured threshold power can be as low as $0.78 \pm 0.1 \mathrm{~mW}$ (see SI Fig. $\mathbf{S 3}$ ), as it is scaled by the free spectral range (FSR) of the resonator:

$$
\mathrm{P}_{\mathrm{th}}=\frac{\pi}{8} \frac{\mathrm{n}}{\mathrm{n}_{2}} \frac{v_{0}}{v_{\mathrm{FSR}}} \frac{\mathrm{A}_{\mathrm{eff}}}{\mathrm{Q}_{\mathrm{i}}^{2}} \times \frac{(1+\kappa)^{3}}{\kappa}
$$

201 where $\mathrm{n}$ is the linear, $\mathrm{n}_{2}$ is the nonlinear refractive index, $v_{0}$ is the pump frequency $v_{\mathrm{FSR}}$ is FSR of the resonator, $\mathrm{A}_{\mathrm{eff}}$ is the effective mode area of the resonator, the coupling factor $\kappa=\kappa_{\mathrm{ex}} / \kappa_{\mathrm{i}}=\mathrm{Q}_{\mathrm{i}} / \mathrm{Q}_{\mathrm{c}}, \kappa_{\mathrm{ex}}$ is the coupling rate, and $\kappa_{\mathrm{i}}$ is the intrinsic rate of the resonator, respectively. Given that $\mathrm{n}^{2}$ has been experimentally measured to be $1.3 \times 10^{-18} \mathrm{~m}^{2} / \mathrm{W}$ (which 205 is almost five times higher than that of $\mathrm{Si}_{3} \mathrm{~N}_{4}$ ), we noticed that the calculated threshold power is in good agreement with the measured value, namely the estimated power is 0.72 $\mathrm{mW}$ (TE $00,20 \mu \mathrm{m}$-radius), $1.4 \mathrm{~mW}$ (TE $00,100 \mu \mathrm{m}$-radius) and $3.4 \mathrm{~mW}$ (TM00, $100 \mu \mathrm{m}-$ radius), respectively. 

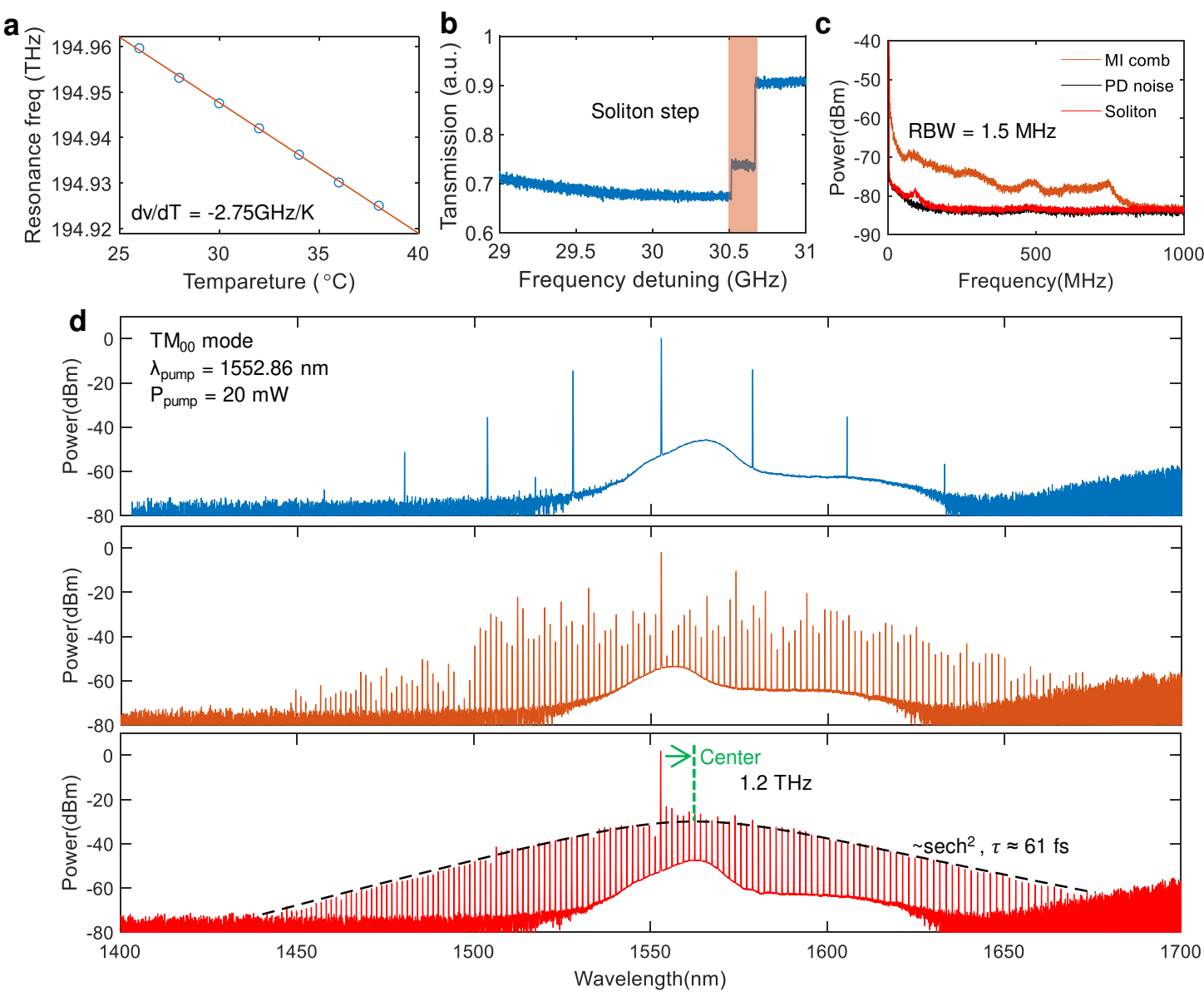

Fig.4 Bright soliton comb generation in a GeSbS microresonator. (a) Measured resonator frequency shift versus temperature for determining TOC of GeSbS resonators. (b) Transmission spectrum of the resonance when a laser is swept. The "soliton step" can be observed, indicating a transition to soliton state. (c) Intensity noise spectrum of MI comb state and soliton states displayed in an electrical spectrum analyzer (ESA).

(d) Mode-locked bright soliton comb generation as the pump laser is red-detuned into the cavity resonance (top to bottom), the pump power is fixed at ca. $20 \mathrm{~mW}$. The single soliton was fitted with a sech ${ }^{2}$ envelope (dashed dark line), indicating an estimated duration of 61 fs. The green arrow shows Raman-induced redshift of the soliton spectrum concerning the pump line.

\section{Generation of soliton microcombs in GeSbS microresonators}

We measured the mode-locked soliton comb operations to demonstrate the advance of our GeSbS platform for microcomb formations. Because high thermo-optical instability is challenging for accessing soliton in resonators ${ }^{35,50}$, a lower TOC $(\mathrm{dn} / \mathrm{dT}$, where $\mathrm{T}$ is temperature) is beneficial. We measured the thermal-optic shift of the resonance frequency when heating the entire chip, see Fig. 4a. From the fitting of temperature-frequency data, 
we determined TOC utilizing the equation:

$$
\frac{\mathrm{dn}}{\mathrm{dT}} \approx-\frac{\mathrm{n}}{\mathrm{v}} \frac{\mathrm{d} v}{\mathrm{dT}} \approx 3.1 \times 10^{-5} \mathrm{~K}^{-1}
$$

Such a TOC value is comparable to that of $\operatorname{Si}_{3} \mathrm{~N}_{4}\left(2.4 \times 10^{-5} \mathrm{~K}^{-1}\right)$ and is around one order of magnitude lower than that of AlGaAs $\left(3.6 \times 10^{-4} \mathrm{~K}^{-1}\right)$ and GaN $\left(\right.$ ca. $\left.10^{-4} \mathrm{~K}^{-1}\right)$. We applied the laser tuning method to form the soliton microcomb. The experimental setup for soliton generation and characterization is shown in SI Fig. S4. During the laser scan process, primary comb separated by 16-FSR $(\mathrm{FSR}=197 \mathrm{GHz})$ and modulation instability (MI) combs were first observed when the pump light was located at the blue-detuned side of the resonance (see Fig. 4d, top and middle). A "soliton step" was observed when the laser was tuned to the red-detuned side, indicating the formation of the dissipative solitons in the cavity $^{51}$, see Fig. 4b. By stopping the laser frequency on the step and slightly adjusting the detuning, a single-soliton-state microcomb was observed with a wavelength span from 1440 $\mathrm{nm}$ to $1680 \mathrm{~nm}$ (see Fig. 4d, bottom). Simultaneously, the drastic reduction of intensity noise of output light shows the transition to a soliton regime with low noise (see Fig. 4c). The soliton duration can be estimated from its $3-\mathrm{dB}$ bandwidth as $61 \mathrm{fs}$ by fitting the final spectrum of a single soliton state with a sech ${ }^{2}$ envelope. We also observed the Raman effectinduced soliton red spectral shift (ca. 1.2 THz in the present case) concerning the pump line (the green arrow in Fig. 4d $)^{52}$. As a result, the lower TOC coefficient and low comb operation power persuade the stable soliton microcombs generations in our GeSbS microresonator without complex laser tuning schemes or auxiliary lasers ${ }^{24}$. 

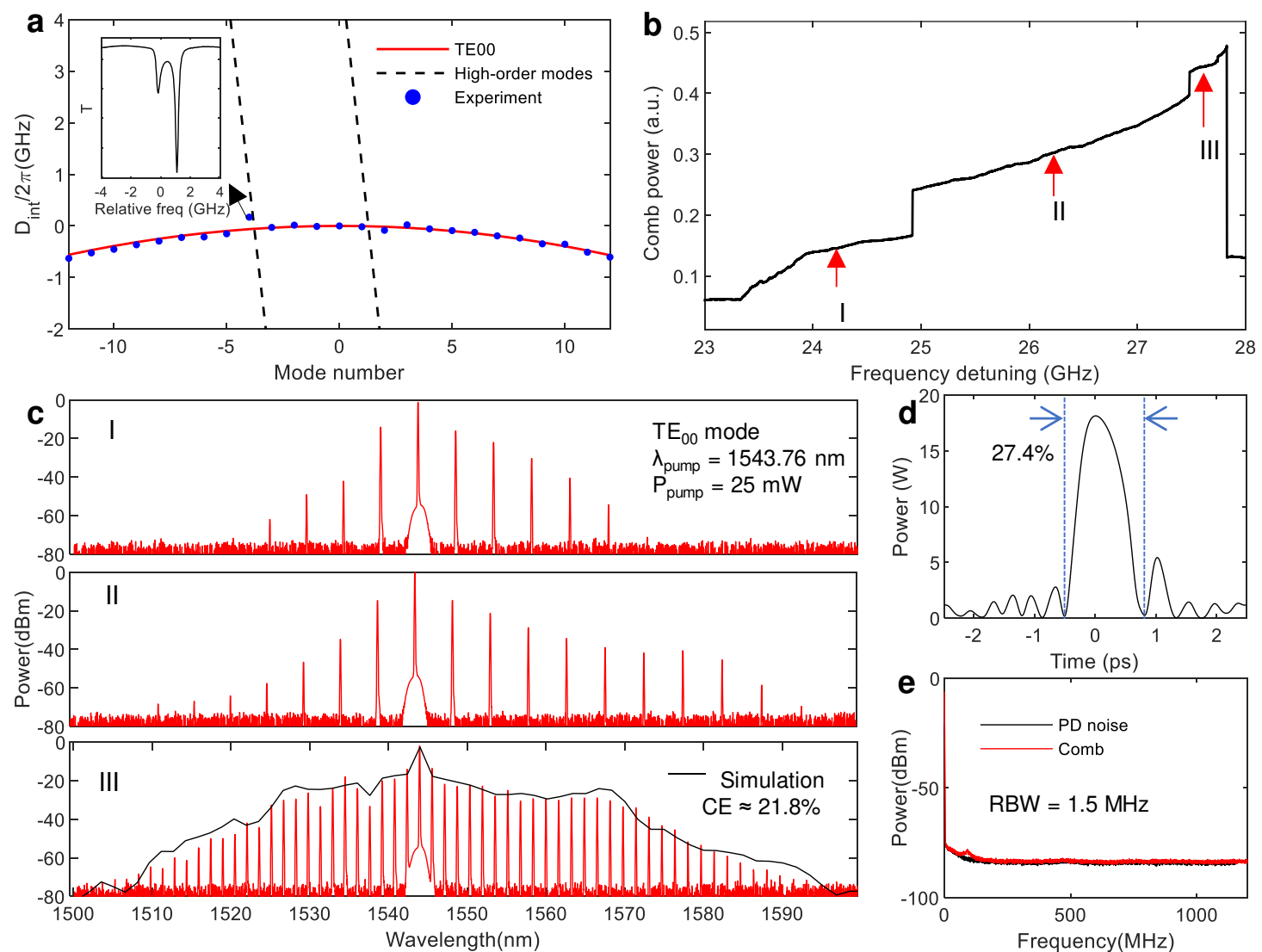

Fig.5 Dark-pulse comb generation in a GeSbS microresonator. (a) Mode structure of the same GeSbS microresonator in $\mathrm{TE}_{00}$ mode. Solid and dashed lines show the simulated integrated dispersion for $\mathrm{TE}_{00}$ and highorder modes. Blue circles represent the measured integrated dispersion of the $\mathrm{TE}_{00}$ mode. The overall mode displays normal dispersion, $\mathrm{D}_{2} / 2 \pi=-8.23 \mathrm{MHz}$, while at $\mu=-4$ (corresponding to ca. $1543 \mathrm{~nm}$ ), the dispersion changes locally to form the mode crossing. Inset: Zoom-in transmission spectrum at $\mu=-4$. (b) Normalized comb power when the laser was scanned from the blue side to the red side. (c) The measured output optical spectra of dark-pulse combs at different stages as indicated in (b), black line denotes the simulated spectrum in stage III. (d) Simulated time-domain intensity profile, showing a dark pulse property and the duty cycle of the solitary wave is ca. $27.4 \%$. (e) Low-intensity noise spectrum of the microcomb measured in a photodiode.

Moreover, given that the $\mathrm{TE}_{00}$ mode family is in the normal dispersion regime, we

further test the possibility of dark pulse-based comb generation in the GeSbS microresonator. Compared with the bright soliton comb in the anomalous dispersion regime, the dark-pulse comb could feature a higher conversion efficiency, while its generation usually requires a localized anomalous effect to initiate the sideband comb modes. By careful analysis of our microresonators, we notice that there exist several localized mode 
calculations, by the coupling between the fundamental $\mathrm{TE}_{00}$ mode (solid line) and highorder modes (dashed lines), see Fig. 5a. The mode coupling would lead to avoided mode crossings (AMXs) on the mode spectrum and introduce localized anomalous effect to the mode where the coupling occurs ${ }^{1}$. In this way, we may choose to pump an AMX mode for dark-pulse comb generation using the laser tuning scheme.

In experiment, the $\mathrm{TE}_{00}$ mode of the microresonator shows an overall normal GVD of $\mathrm{D}_{2} / 2 \pi=-8.23 \mathrm{MHz}$, and the selected AMX mode is at $1543 \mathrm{~nm}$. The comb generation was observed by manually tuning the laser from the blue side to the red side of the resonance when the pump laser was launched into the bus waveguide with $25 \mathrm{~mW}$ (see Fig. 5b). The comb power trace shows three steps during the laser tuning process, associated with the different states of the microcomb in normal dispersion region ${ }^{53}$. Figure $\mathbf{5 c}$ shows the evolution of the output optical spectra. At stage I, 3-FSR spaced comb lines were initially observed. Next to stage II, the bandwidth was increased with further tuning the pump frequency, and a flat wing at $1578 \mathrm{~nm}$ was formed. Finally, when the pump laser was stopped at stage III, we obtained the microcomb covering the range from $1510 \mathrm{~nm}$ to 1590 $\mathrm{nm}$ with a repetition rate of ca. $200 \mathrm{GHz}$. As a critical factor, the measure power conversion efficiency $\left(\mathrm{CE}=\mathrm{P}_{\text {other }}^{\text {out }} / \mathrm{P}_{\text {pump }}^{\text {in }}\right.$, where $\mathrm{P}_{\text {other }}^{\text {out }}$ is the sum of all comb lines excluding the pump and $\mathrm{P}_{\text {pump }}^{\text {in }}$ is the input power on-chip) is around $21.8 \%$, which could be improved by optimizing the coupling structure or introducing coupled-microresonator geometry for dynamic adjustment of AMXs based on our GeSbS ChG platform ${ }^{8}$.

Meanwhile, we performed numerical simulations based on the Lugiato-Lefever equation (LLE) model $^{54}$. As a result, the simulated waveform is in good agreement with the 
experimental result, see Fig. 5c and 5d. In addition, the duty cycle of the temporal darkpulse pattern is simulated to be around $27.4 \%$, which is also close to the measured conversion efficiency ${ }^{54}$, see Fig. 5d. Moreover, the comb was assessed on the lowfrequency spectrum in the radio-frequency (RF) domain and showed a low-noise figure.

\section{Conclusion}

We have presented an integrated nonlinear photonics platform for soliton microcombs based on our home-developed GeSbS microresonators, which featured high $Q$-factor up to ca. $2.3 \times 10^{6}$, higher nonlinearity, low TOC, and high LDT. The OPO pump threshold as low as $780 \mu \mathrm{W}$ was attained. We achieved completely different dispersion curves by precise 
evaporation method was used to deposit the $\mathrm{Ge}_{20} \mathrm{Sb}_{10} \mathrm{~S}_{65}$ film on $\mathrm{Si}$ substrates with a $3 \mu \mathrm{m} \mathrm{SiO}_{2}$ layer in a vacuum chamber at a base pressure of $7 \times 10^{-6} \mathrm{~Pa}$. The substrates were mounted on a rotatable hold and pretreated using Ar plasma to improve the adhesion between the films and substrates. The evaporation rate was set to approximately $5-6 \mathrm{~nm} / \mathrm{min}$.

Microresonator fabrication. As illustrated in Fig. 2a, a layer of GeSbS $(850 \mathrm{~nm})$ was deposited on top of $\mathrm{Si}$ substrate with a $3 \mu \mathrm{m} \mathrm{SiO}{ }_{2}$ layer. The chip was put into a vacuum furnace and processed at $350{ }^{\circ} \mathrm{C}$. It was then coated with a photoresist (ARP-6200) with a thickness of ca. $800 \mathrm{~nm}$ and patterned by electron beam lithography (EBL). After development, a thermal reflow process was applied on a hotplate at $140{ }^{\circ} \mathrm{C}$ for 3 mins to remove the roughness of the sidewalls of the patterns. $\mathrm{RIE}$ with $\mathrm{CF}_{4} / \mathrm{CHF}_{3} / \mathrm{Ar}$ gas chemistries was then applied to transfer the patterns to the GeSbS layer. Afterward, an ICP-RIE was used to remove the residual resist. Finally, the sample was clad with 3- $\mu$ m-thick $\mathrm{SiO}_{2}$ by ICP-CVD deposition at $300^{\circ} \mathrm{C}$. The inverse tapers and microresonator were simultaneously prepared under the same fabrication conditions, achieving the lowest coupling loss of $-2.3 \mathrm{~dB}(45 \%)$ per facet at $1550 \mathrm{~nm}$.

Characterizations of LDT, $\mathbf{Q}$ factor, dispersion-engineering, and comb generations. The LDT was measured using a similar method ${ }^{56}$ that the $\mathrm{Ge}_{20} \mathrm{Sb}_{10} \mathrm{~S}_{65}$ films were shined with a femtosecond laser from an optical parametric amplifier (Coherent, Opera-HP) with a repetition rate of $10 \mathrm{kHz}$, a center wavelength of $1550 \mathrm{~nm}$, and a pulse width of $250 \mathrm{fs}$. The laser was focused on a spot size of $200 \mu \mathrm{m}$ on the top surface of the $\mathrm{ChG}$ film by a $\mathrm{CaF}_{2}$ lens with a focal length of $50 \mathrm{~mm}$. The temperature was stabilized at $30^{\circ} \mathrm{C}$ to minimize the temperature drift-induced resonance shift. A continuous-wave tunable laser (Keysight 81606A) was used to characterize the linear properties of microresonators. optical coupler with a splitting ratio of 90:10, and a fiber loop with a length of $\sim 10 \mathrm{~m}$ was utilized to 
calibrate the frequency difference ${ }^{21}$. The probe light was divided into two channels (passing through the GeSbS resonator and the fiber ring resonator, respectively). Then, they were received by two photodetectors (New Focus, Model 1811FC) monitored by an oscilloscope (Keysight, DSOS404A) to record transmission traces. was amplified by an erbium-doped fiber amplifier (EDFA) (Amonics, AEDFA-33-B-FA), the output light was split into three parts, see SI Fig. S4. One was recorded by an optical spectrum analyzer (OSA) (YOKOGAWA AQ6370D). The second was detected by a photodetector, which was monitored by an electrical spectrum analyzer (ESA) (Agilent N9030A) for determining the intensity noise of the generated comb. The third part was received by a photodetector, which was monitored by the oscilloscope for recording the power trace of pump light.

\section{Supporting Information}

Supporting information is available from the corresponding author upon reasonable request.

\section{Acknowledgments}

National Key R\&D Program of China under Grant (2019YFA0706301), Key Project in

Broadband Communication and New Network of the Ministry of Science and Technology

(MOST) (2018YFB1801003), National Science Foundation of China (NSFC) (U2001601, 
350 Author contributions

351 D Xia, P Zeng, Z Yang, J Wu, B Zhang and H Guo conceived the experiment, D Xia, Z

352 Wang, L Luo' did the simulation and design for the devices. D Xia, J Wu, J Huang, Z Wang 353 and B Zhang performed the experiments. P Zeng, Z Yang, Mi Gao, D Liu, and S Yang did 354 the fabrication. D Xia P Zeng, Z Yang, J Wu, B Zhang and H Guo analyzed the data. B 355 Zhang and Z Li supervised this project. All the authors contributed in writing the manuscript.

\section{Conflict of Interest}

357 The authors declare no conflict of interests. 


\section{References:}

1. Xue X, et al. Mode-locked dark pulse Kerr combs in normal-dispersion microresonators. Nat Photonics 9, 594-600 (2015).

2. He M, et al. High-performance hybrid silicon and lithium niobate Mach-Zehnder modulators for $100 \mathrm{Gbit} \mathrm{s}^{-1}$ and beyond. Nat Photonics 13, 359-364 (2019).

3. Spencer DT, et al. An optical-frequency synthesizer using integrated photonics. Nature 557, 81-85 (2018).

4. Eggleton BJ, et al. Brillouin integrated photonics. Nat Photonics 13, 664-677 (2019).

5. Yang KY, et al. Bridging ultrahigh-Q devices and photonic circuits. Nat Photonics 12, $297-$ 302 (2018).

6. Liu J, et al. High-yield, wafer-scale fabrication of ultralow-loss, dispersion-engineered silicon nitride photonic circuits. Nat Commun 12, 2236 (2021).

7. Gaeta AL, et al. Photonic-chip-based frequency combs. Nat Photonics 13, 158-169 (2019).

8. Guidry MA, et al. Quantum optics of soliton microcombs. Nat Photonics 16, 52-58 (2022).

9. Chang L, et al. Ultra-efficient frequency comb generation in AlGaAs-on-insulator microresonators. Nature Communications 11, 1-8 (2020).

10. Pfeifle J, et al. Coherent terabit communications with microresonator Kerr frequency combs. Nat Photonics 8, 375-380 (2014).

11. Corcoran B, et al. Ultra-dense optical data transmission over standard fibre with a single chip source. Nat Commun 11, 2568 (2020).

12. $\mathrm{Hu} \mathrm{H}$, et al. Single-source chip-based frequency comb enabling extreme parallel data transmission. Nat Photonics 12, 469-473 (2018).

13. Marpaung D, et al. Integrated microwave photonics. Nat Photonics 13, 80-90 (2019).

14. Riemensberger J, et al. Massively parallel coherent laser ranging using a soliton microcomb. Nature 581, 164-170 (2020).

15. Liu J, et al. Monolithic piezoelectric control of soliton microcombs. Nature 583, 385-390 (2020).

16. Merklein M, et al. A chip-integrated coherent photonic-phononic memory. Nat Commun 8, 574 (2017).

17. Tan T, et al. Multispecies and individual gas molecule detection using Stokes solitons in a graphene over-modal microresonator. Nat Commun 12, 6716 (2021).

18. Tian H, et al. Magnetic-free silicon nitride integrated optical isolator. Nat Photonics 15, 828836 (2021).

19. Yao B, et al. Gate-tunable frequency combs in graphene-nitride microresonators. Nature $\mathbf{5 5 8}$, 410-414 (2018).

20. Stern B, et al. Battery-operated integrated frequency comb generator. Nature 562, 401-405 (2018).

21. Wang W, et al. Self-locked orthogonal polarized dual comb in a microresonator. Photon Res $\mathbf{6}$, 363-367 (2018).

22. Liu X, et al. Aluminum nitride nanophotonics for beyond-octave soliton microcomb generation and self-referencing. Nat Commun 12, 5428 (2021).

23. Weng $\mathrm{H}$, et al. Directly accessing octave-spanning dissipative Kerr soliton frequency combs in an AlN microresonator. Photonics Research 9, 1351-1357 (2021).

24. Zheng Y, et al. Integrated Gallium Nitride Nonlinear Photonics. Laser \& Photonics Rev 16, 
2100071 (2021).

25. Yu M, et al. Raman lasing and soliton mode-locking in lithium niobate microresonators. Light Sci \& Appl 9, 9 (2020).

26. Wu R, et al. Lithium niobate micro-disk resonators of quality factors above 10^7. Opt Lett $\mathbf{4 3}$, 4116-4119 (2018).

27. Fang Z, et al. Efficient electro-optical tuning of an optical frequency microcomb on a monolithically integrated high-Q lithium niobate microdisk. Opt Lett 44, 5953-5956 (2019).

28. Wang $\mathrm{C}$, et al. High-Q microresonators on $4 \mathrm{H}$-silicon-carbide-on-insulator platform for nonlinear photonics. Light Sci \& Appl 10, 139 (2021).

29. Moille G, et al. Dissipative Kerr Solitons in a III-V Microresonator. Laser \& Photonics Rev 14, 2000022 (2020).

30. Zhang $\mathrm{M}$, et al. Broadband electro-optic frequency comb generation in a lithium niobate microring resonator. Nature 568, 373-377 (2019).

31. Shen B, et al. Integrated turnkey soliton microcombs. Nature 582, 365-369 (2020).

32. Raja AS, et al. Electrically pumped photonic integrated soliton microcomb. Nature Communications 10, 1-8 (2019).

33. Xiang C, et al. Laser soliton microcombs heterogeneously integrated on silicon. Science 373, 99-103 (2021).

34. Ji X, et al. Ultra-low-loss on-chip resonators with sub-milliwatt parametric oscillation threshold. Optica 4, 619-624 (2017).

35. Jung H, et al. Tantala Kerr nonlinear integrated photonics. Optica 8, 811-817 (2021).

36. Liang Q, et al. Ultrasensitive multispecies spectroscopic breath analysis for real-time health monitoring and diagnostics. Proc Natl Acad Sci 118, e2105063118 (2021).

37. Eggleton BJ, et al. Chalcogenide photonics. Nat Photonics 5, 141-148 (2011).

38. Lin H, et al. Chalcogenide glass-on-graphene photonics. Nat Photonics 11, 798-805 (2017).

39. Gao J, et al. Near-infrared to ultra-violet frequency conversion in chalcogenide metasurfaces. Nat Commun 12, 5833 (2021).

40. Yu Y, et al. A broadband, quasi-continuous, mid-infrared supercontinuum generated in a chalcogenide glass waveguide. Laser \& Photonics Rev 8, 792-798 (2014).

41. Zhao Z, et al. Mid-infrared supercontinuum covering 2.0-16 $\mu \mathrm{m}$ in a low-loss telluride singlemode fiber. Laser \& Photonics Rev 11, 1700005 (2017).

42. Petersen CR, et al. Mid-infrared supercontinuum covering the 1.4-13.3 $\mu \mathrm{m}$ molecular fingerprint region using ultra-high NA chalcogenide step-index fibre. Nat Photonics 8, 830-834 (2014).

43. Li L, et al. Integrated flexible chalcogenide glass photonic devices. Nat Photonics 8, 643-649 (2014).

44. Morrison B, et al. Compact Brillouin devices through hybrid integration on silicon. Optica 4, 847-854 (2017).

45. Kim DG, et al. Universal light-guiding geometry for on-chip resonators having extremely high Q-factor. Nat Commun 11, 5933 (2020).

46. Wang T, et al. Systematic z-scan measurements of the third order nonlinearity of chalcogenide glasses. Optical Materials Express 4, 1011-1022 (2014).

47. Xia D, et al. Integrated Ge-Sb-S chalcogenide microresonator on chip for nonlinear photonics. In: Conference on Lasers and Electro-Optics/Pacific Rim ( $\mathrm{ed}^{\wedge}(\mathrm{eds})$. Optical Society of America 
(2020).

448 48. Wang W, et al. Advances in soliton microcomb generation. Advanced Photonics 2, 034001 449 (2020).

450 49. Brasch V, et al. Photonic chip-based optical frequency comb using soliton Cherenkov radiation. 451 Science 351, 357-360 (2015).

452 50. Jiang X, et al. Optothermal dynamics in whispering-gallery microresonators. Light Sci \& Appl 453 9, 1-15 (2020).

454 51. Guo H, et al. Universal dynamics and deterministic switching of dissipative Kerr solitons in 455 optical microresonators. Nature Physics 13, 94-102 (2017).

456 52. Karpov M, et al. Raman self-frequency shift of dissipative Kerr solitons in an optical 457 microresonator. Phys Rev Lett 116, 103902 (2016).

458 53. Godey C, et al. Stability analysis of the spatiotemporal Lugiato-Lefever model for Kerr optical 459 frequency combs in the anomalous and normal dispersion regimes. Phys Rev A 89, 21 (2014).

460 54. Xue X, et al. Microresonator Kerr frequency combs with high conversion efficiency. Laser \& 461 Photonics Rev 11, (2017).

462 55. Zhang B, et al. High brightness $2.2-12 \mu \mathrm{m}$ mid-infrared supercontinuum generation in a 463 nontoxic chalcogenide step-index fiber. J Am Ceram Soc 99, 2565-2568 (2016).

464 56. Shang H, et al. On-Chip Detector Based on Supercontinuum Generation in Chalcogenide 465 Waveguide. J Lightwave Technol 39, 3890-3895 (2021). 


\section{Supplementary Files}

This is a list of supplementary files associated with this preprint. Click to download.

- Supplementaryinformation.pdf 